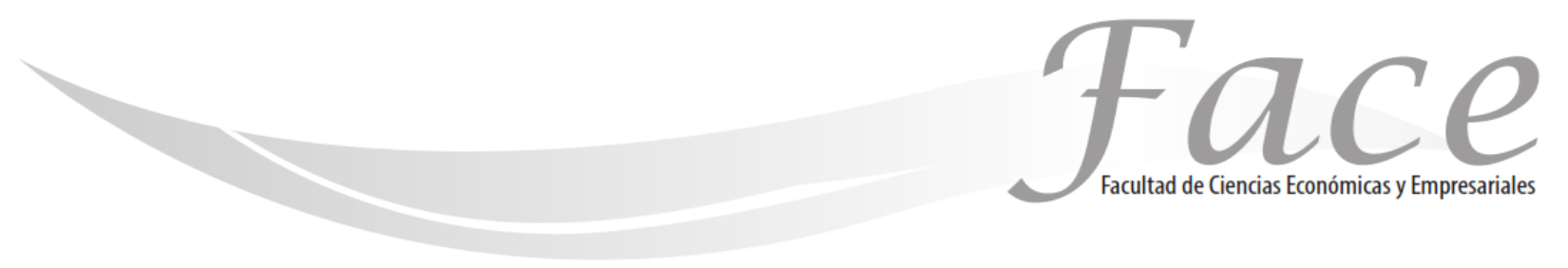

ISSN Impreso: 1794-9920

ISSN Electrónico: 2500-9338

Volumen $16-\mathrm{N}^{\circ} 2$

Año 2016

Págs. 5 - 19

\title{
TRASTORNOS DE PERSONALIDAD Y VIOLENCIA. ARTÍCULO DE REFLEXIÓN PARA EL POSCONFLICTO COLOMBIANO
}

Óliver Lis *

Enlace ORCID: http://orcid.org/0000-0001-7495-0314

Fecha de Recepción: 17 de Marzo 2016

Fecha de Aprobación: 18 de Junio 2016

\begin{abstract}
Resumen:
El presente trabajo, procura revisar toda la literatura científica a disposición sobre la relación entre algunos trastornos de personalidad y violencias, cuyo análisis, hace parte de la epistemología necesaria para el desarrollo de políticas adecuadas para el posconflicto colombiano, con especial atención a la búsqueda, de garantías de no-repetición, ideal consignado por el Estado colombiano, en la ley 1448 de 2011 de Reparación de Víctimas. El autor consultó 485 artículos de las bases de datos científicas: Lilacs, Sdici, ScienceDirect, PubMed, Sociel Sciences Citation Index, extrayendo solo unas decenas pertinentes para ocuparse de la relación entre trastornos de personalidad (psicopatía, trastorno disocial, trastorno antisocial, trastorno negativista desafiante, trastorno límite de la personalidad) y violencias (especialmente la violencia armada) desde una atalaya científica, aproximándose así a las causas psicológicas del conflicto armado colombiano y demás formas de violencia, esbozando la necesidad de políticas para hacer frente a este fenómeno y de persistir y reforzar en esa línea investigativa.
\end{abstract}

Palabras Claves: Trastornos de personalidad, Violencia, Posconflicto Colombiano, Colombia. Psicopatía, trastorno disocial, trastorno antisocial, trastorno negativista desafiante, trastorno límite de la personalidad.

* Óliver Lis. Psicólogo de la Universidad Surcolombiana, en proceso de aceptación como maestrante para Salud Mental Forense en la Universidad Nacional de La Plata, Argentina. Actualmente editor en Jefe - CEO para Editorial Popayán, División de Proyectos Especiales de Indugráfica de Occidente Ltda. y editor asociado de la Fundación Caucana de Patrimonio Intelectual, Popayán - Colombia. Correo electrónico: oliverlicht@gmail.com 

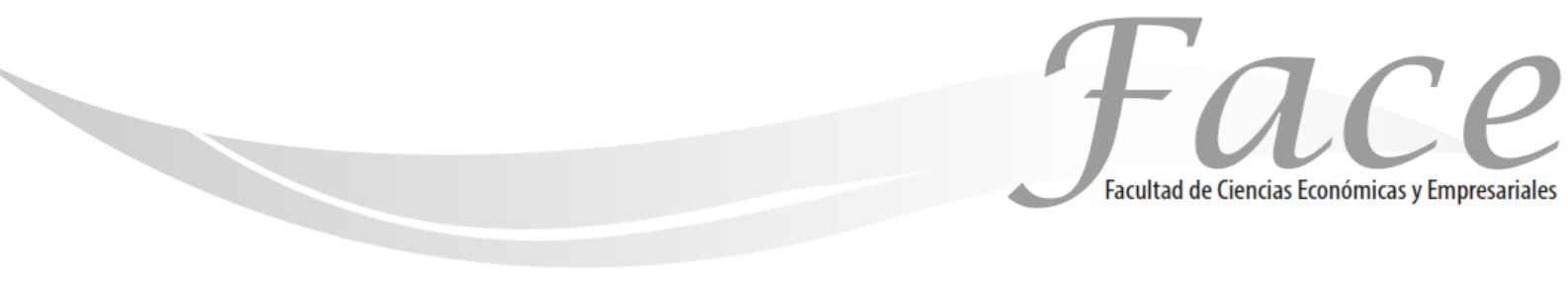

\title{
PERSONALITY DISORDERS AND VIOLENCE. ARTICLE OF REFLECTION FOR THE COLOMBIAN POST-CONFLICT.
}

\begin{abstract}
:
The present work aims to review all scientific literature available on the relationship between some personality disorders and violence, whose analysis is a part of the necessary epistemology for the development of appropriated policies for the Colombian post-conflict, with particular attention to the search of guarantees of non-repetition, ideal consigned by the colombian State, in the law 1448 of 2011 about Victims Reparation. the author reviewed 485 items of scientific databases: Lilacs, Sdici, ScienceDirect, PubMed, Sociel Sciences Citation Index, extracting only a relevant dozens to deal with the relationship between personality disorders (psychopathy, conduct disorder, antisocial disorder , oppositional defiant disorder, borderline personality disorder) and violences (especially armed violence) from a scientific vantage point, thus approaching to the psychological causes of the colombian armed conflict and other forms of violence, outlining the necessity for policies to dealing with this phenomenon and persisting, and reinforcing this research line.
\end{abstract}

Keywords: Personality disorders, Violence, Colombian Postconflict, Colombia, Psychopathy, conduct disorder, antisocial personality disorder, oppositional defiant disorder, borderline personality disorder.

\section{TRANSTORNOS DE PERSONALIDADE E VIOLÊNCIA. ARTIGO DE REFLEXÃO PARA O PÓS- CONFLITO COLOMBIANO.}

\section{Resumo:}

Este trabalho tem como objetivo revisar toda a literatura científica disponível sobre a relação entre alguns transtornos de personalidade e violência, cuja análise é parte da epistemologia necessário para o desenvolvimento de políticas adequadas para o pós-conflito colombiano, com especial atenção para a busca, garantias de não repetição, ideal expedido pelo Estado colombiano, na lei 14482011 Vítimas Reparação. 0 autor consultou 485 itens de bases de dados científicos: Lilacs, Sdici, ScienceDirect, PubMed, sociel Sciences Citation Index, extraindo apenas dezenas relevantes para lidar com a relação entre transtornos de personalidade (psicopatia, transtorno de conduta, transtorno anti-social, transtorno de oposição desafiante, transtorno de personalidade borderline) e violência (especialmente a violência armada) a partir de um ponto de vista científico, aproximando-se assim as causas psicológicas do conflito armado colombiano e outras formas de violência, destacando a necessidade de políticas para resolver este fenómeno e persistir e reforçar esta linha de pesquisa.

Palavras-Chave: Trastornos de personalidad, Violencia, Posconflicto Colombiano, Colombia. Psicopatía, trastorno disocial, trastorno antisocial, trastorno negativista desafiante, trastorno límite de la personalidad. 


\section{INTRODUCCIÓN:}

La carga mundial de la enfermedad (AVAD) de trastornos mentales entre las enfermedades no transmisibles es del $28 \%$ sin descarte de comorbilidad violenta (Prince et al., 2007). El costo de los trastornos mentales en Europa fue de 523,3 mil millones de euros en 2010 según Gustavsson et al. (1). Este es un problema grave de salud pública, que requiere de atención y acción urgente en nuestro medio.

Existe afortunadamente un reconocimiento por parte de las potencias mundiales acerca de la necesidad de hacer todo lo urgente y lo necesario en este sentido, para entender y controlar dicho fenómeno: El presidente Obama impulsó BRAIN (la investigación sobre el cerebro humano más costosa de la historia, mediante la mejora innovadora de neurotecnologías); a partir de mayo 2013, también se dio origen en la U.E. al mes del Cerebro promoviendo debates sobre las inversiones en la excelencia científica y estrategias para aumentar el beneficio del paciente y la prevención de las enfermedades neuropsicológicas.

Lograr esa comprensión de la relación entre estos trastornos de personalidad con las violencias, es uno de los desafíos identificados en "Horizonte 2020", programa marco de investigación e innovación de la Comisión Europea (2014-2020), así como dentro de las estrategias de investigación presentadas por otras agencias de financiación según Schumanna, et. al., (2).

A medida que se avanza hacia la comprensión de los trastornos de personalidad para que sean estos tratados como enfermedades, es descorrido un antiguo velo de incertidumbre acerca de otras ciencias que se han empeñado inútilmente en desentrañar las causas o la etiología de la violencia armada, pero igualmente importantes para la comprensión global del fenómeno.

Con base en la entrevista diagnóstica compuesta o "CIDI2" realizada a partir de criterios diagnósticos tomados del "Manual Diagnóstico y Estadístico de los Trastornos Mentales, versión IV (DSM IV)", entrevista aplicada por la Organización Mundial de la Salud (OMS), Colombia presentó en 1997 una prevalencia de al menos un trastorno psiquiátrico para el $30 \%$ de su población (Cifras del Minsalud, 1997).

En 2003, se reportó una prevalencia del $40,1 \%$ con ausencia de tratamiento médico psiquiátrico el año previo al estudio realizado.
La última investigación epidemiológica realizada por la OMS estimó que para el año 2020 Colombia contará con una de las prevalencias de trastornos de personalidad y trastornos mentales, más altas del mundo (Ministerio de Salud y Colciencias, ENSM, 2015).

En, 1992 la OMS publicó también unos Criterios Diagnósticos para el Trastorno Disocial de la Personalidad, que incluyen una cruel despreocupación por los sentimientos de los demás y falta de empatía; una actitud marcada y persistente de irresponsabilidad y despreocupación por las normas, reglas y obligaciones sociales; la inhabilidad para mantener relaciones personales duraderas; una muy baja tolerancia a la frustración, o un bajo umbral para evitar las descargas de agresividad, dando lugar a comportamientos violentos; con la incapacidad subsiguiente de sentir culpa y de aprender de la experiencia, en particular de los castigos (ineficacia del propósito rehabilitador de las prisiones); una marcada predisposición a ofrecer racionalizaciones verosímiles del comportamiento conflictivo culpando a los demás; irritabilidad persistente, entre otras.

De manera coherente con este empeño de formular unos criterios por parte de la OMS para establecer este trastorno que como se verá, hace parte de la psicopatía, trastorno de personalidad que compromete violencia, la Asociación Americana de Psiquiatría publicó en su cuarta versión del DSM, otros criterios comunes, como un patrón general de desprecio y violación de los derechos de los demás que se presenta a partir de la edad de 15 , con presencia de tres $(0$ más) de los siguientes ítems: Fracaso para adaptarse a las normas sociales en lo que respecta al comportamiento legal (perpetración de repetida de actos que son motivo de detención), deshonestidad, mentiras reiterativas, estafa a otros para obtener un beneficio personal o por placer, impulsividad, irritabilidad y agresividad, peleas físicas repetidas 0 agresiones; despreocupación imprudente por su seguridad o la de los demás; irresponsabilidad persistente, indicada por la incapacidad de mantenerse en un trabajo con constancia o de hacerse cargo de obligaciones económicas; falta de remordimientos, indiferencia o justificación de haber cometido daño, maltrato o robado a otros, diagnóstico que cada vez deja de circunscribirse a pruebas psicométricas para ser confirmado por pruebas de cariotipo y neuropsicológicas, como las de Turpin, et. al. (3); Blancharda, Lyonsa, et. al. (4), Morris, Skuse et al. (5), Lake, Baksh, et al. (6), Kessler \& Moos (7). 
Inicialmente, se consideró la posibilidad de hacer un metaanálisis de todos los artículos científicos que estudian la relación entre trastornos de personalidad (como psicopatía, trastorno disocial, trastorno antisocial, trastorno negativista desafiante, trastorno límite de la personalidad) y las diversas formas de violencia (incluyendo la armada, de género, intrafamiliar, económica, social, psicológica), evidenciando: primero, la ausencia de un instrumento estandarizado para tal propósito como una batería universal o una escala de medición universal capaz de hacer viable la estandarización de resultados; dificultad que se presenta, incluso al abordar un mismo trastorno de personalidad.

De contar los científicos con dicha escala universal, sería viable un análisis multifactorial de los comportamientos violentos (en tanto efectos) y de sus factores etiológicos, al menos, dentro de una misma tipología de diferentes trastornos.

Su ausencia crea una primera necesidad que es la de hacer una estandarización universal de criterios diagnósticos, de criterios psicopatológicos para tener un instrumento base capaz de identificar la prevalencia de estos elementos que componen tales trastornos de personalidad que pueden desembocar en expresiones violentas.

Así pues, entre 485 artículos científicos revisados sobre trastornos de personalidad y diversas formas de violencia consultados en diversas bases de datos, no fue posible dar cuenta de una tipología universal que permitiese metaanalizar las conductas de los distintos agresores o su tratamiento; tampoco ha respondido a unos mismos estándares científicos, lo cual imposibilitó generar un metaanálisis, lo que limita a un artículo de reflexión el procedimiento.

Como segundo punto: no hay una semiótica universal para la etiología de muchos de estos problemas, por el hecho mismo de que son trastornos de personalidad y no enfermedades (las cuales presentan un claro origen mental, genómico, neurológico, psicológico, o social, etc.).

De reconocerse por la ciencia un agente etiológico común con síntomas, signos y alteraciones claramente identificables, se podría hablar acerca de una enfermedad y no de trastornos.

Para fines de lograr la mejor comprensión del presente artículo, es necesario hacer una distinción previa entre enfermedad, síndrome y trastorno:

A. ENFERMEDAD. Tiene: 1. Agente etiológico reconocible, 2. grupo identificable de signos (manifestaciones observables objetivas, claves para el correcto diagnóstico) y síntomas (manifestaciones que solo percibe el paciente por lo que se consideran subjetivas como por ejemplo: el dolor); 3. presentación de alteraciones anatómicas consistentes): Los trastornos de la personalidad no deben confundirse con enfermedades.

B. SÍNDROME. 1. Presenta signos y 2. síntomas con 3. una etiología desconocida, o reacción ante situación vital perturbadora.

C. TRASTORNO. 1. Presenta síntomas asociados a patologías; 2. se caracteriza por alteraciones de los procesos cognitivos y del desarrollo, 3. no tiene una etiología totalmente conocida.

*Algunos trastornos recogen ciertos sindromes (véase el CIE-10).

No ha sido posible razón de estos criterios nosológicos, decir que los trastornos de personalidad que tienen comorbilidad violenta, constituyan una enfermedad, pese a la presencia de un agente etiológico común reconocible, con síntomas, signos y alteraciones claramente identificables.

Se espera que la ciencia avance rápidamente, para realizar un estudio de prevalencia psicopatológica de trastornos de personalidad, aunque esa nosología que hoy se presenta previa o parcialmente, no constituye motivo para desestimar dichos trastornos como un problema grave y dejar de generar políticas y planes de intervención a este respecto.

La presencia de los trastornos de personalidad que comportan violencia en los diferentes manuales de diagnóstico, su reconocimiento en la literatura científica denotan claramente un problema grave que requiere de políticas encaminadas a la generación de una epidemiología que abarque la totalidad de la población.

En aras de aportar a ese proceso, y de dar más razones para comprenderlo desde otra atalaya que ha sido omitida o ajena a las mesas de negociación del conflicto armado colombiano, se presenta éste artículo de reflexión, brindando algunos criterios científicos en una amplia y cuidadosa revisión de la literatura científica.

La ley 1616 de 2013 le da alcances constitucionales al derecho a la salud mental (véase Diario Oficial 48680 de enero 21 de 2013), y también obliga al Estado colombiano a garantizar dicho derecho.

El Plan Decenal de Salud Pública de Colombia (PDSP) 2012-2021 del Gobierno Santos que es el plan marco para todos los proyectos nacionales de salud pública (publicado por primera vez el 22 de mayo de 2012 en la página del 
Ministerio de Salud), contempla un enfoque diferencial de la salud en Colombia.

Otros recursos de los que debería disponer Colombia para tratar el tema de trastornos de personalidad son los del Ministerio del Posconflicto (según puede ser consultado en noticia del 9 de noviembre de 2015 en la web de la Presidencia de la República), con un presupuesto actual que asciende a más de 470 millones de dólares (en noticias de El Espectador del 4 de enero de 2016) para invertir en políticas orientadas al cumplimiento de los ideales de atención a las víctimas de la violencia armada y de la paz.

\section{METODOLOGÍA:}

Es preciso referirse a 10 violento, como expresión lingüística, razón de un espectro de significaciones de la violencia mucho más amplias. El término violencia para efectos de este artículo de reflexión hará alusión exclusivamente a violencias patológicas, entendidas estas últimas como aquellas en las que se ven comprometidos los derechos humanos naturales e inalienables 0 el Derecho Internacional Humanitario, o los Derechos Fundamentales consignados en la Carta Política de Colombia, para descarte de otras que aunque hacen parte del espectro lingüístico de lo violento, no vulneran dichos derechos (garantías que hoy, afortunadamente se extienden a otras especies que gozan de sistemas nerviosos altamente complejos).

Se consultó un total de 485 artículos científicos en castellano e inglés en las bases de datos científicas: Lilacs, Sdici, ScienceDirect, PubMed, Sociel Sciences Citation Index, extrayendo solo un $8,5 \%$ en el que se presentaba una relación entre trastornos de personalidad (psicopatía, trastorno disocial, trastorno antisocial, trastorno negativista desafiante, trastorno límite de la personalidad) y diversas formas de violencia (especialmente la violencia armada); en algunos casos, con un criterio de aproximación nosográfica como "trisomía y psicopatía", "psychopathy and trisomy"; seguimiento no superior a un período de 1 año (de mayo de 2015 a mayo de 2016), sin discriminación alguna sobre la fecha de publicación de los artículos encontrados; esto se confrontó con otros trabajos con el sello de la World Health Organization (WHO).

\section{Revisión de la Literatura Científica: Relación entre Trastornos de Personalidad y Formas de Violencia.}

Como ya se mencionó, fueron consultadas las bases de datos en inglés y castellano sin discriminación sobre las fechas de publicación, y otros trabajos con el sello de la World Health Organization (WHO), presentando una relación con las diferentes formas de violencia los siguientes trastornos (análisis inductivo, artículos científicos-Manuales de Diagnóstico Estadístico):

\section{En el DSM-V:}

"313.81 (F91.3). Trastorno negativista desafiante (243)

"V71.01 (Z72.811) Comportamiento antisocial del adulto.

"V71.02 (Z72.810) Comportamiento antisocial infantil o adolescente.

\begin{tabular}{llllr} 
En el CIE-10 & (clasificación & \multicolumn{2}{l}{ internacional de } \\
enfermedades & 0 & ICD & - & International \\
StatisticalClassification & of & Diseases and \\
RelatedHealthProblems): & & &
\end{tabular}

"F60.2 Trastorno disocial de la personalidad.

Incluye: trastorno de personalidad sociopática, trastorno de personalidad amoral, trastorno de personalidad asocial, trastorno de personalidad antisocial, trastorno de personalidad psicopática.

Excluye: trastornos disociales (F91) trastorno de inestabilidad emocional de personalidad".

\section{Características Nosológicas y Criterios Diferenciales}

Para definir estos trastornos de personalidad, como la psicopatía que es causa de violencia (social, familiar, política, económica y/o armada, etc.), se determinaron alteraciones a diferentes niveles; a saber:

1. Genético, como presencia de alteración genética, trisomía, en lo que normalmente es el cuarto par de cromosomas (con pruebas de cariotipo humano) de acuerdo a Turpin, et. al. (8); Blancharda, Lyonsa, et. al. (9), Morris, Skuse et al. (10), Lake, Baksh, et al. (11), Kessler \& Moos (12).

2. Neurológico, expresión de las alteraciones genéticas descritas en el enunciado anterior, tales como problemas de comunicación anatómico entre la amígdala y la corteza prefrontal (encargada del procesamiento de estímulos, lo que genera impulsividad irrefrenable de conductas sexuales y de agresivas), conllevando a una ausencia total de culpa y empatía, conforme a Vidal (13), Völlm y Taylor et. al. (14); Schumanna, Binderb, et. al. (15) y Hare (16), lo que en otras palabras, quiere decir que estos sujetos, están genética, neurológica y psicológicamente lisiados para experimentar culpa, y ponerse así, en "los zapatos del otro", no como un proceso racional que le es posible y es plenamente consciente del daño que hace, sino como un 
proceso emocional: el psicópata está emocionalmente lisiado según Hare (17).

Para Blair (18), la investigación científica da prelación a factores genéticos en aras de presentar una definición de enfermedad para estos trastornos:

"La psicopatía, conceptualizada como una entidad clínica, es fundamentalmente distinta a una colección heterogénea de síndromes que abarca el término trastorno de conducta. Se procede a proporcionar una cuenta de la evolución de la psicopatía en múltiples niveles: último causal (la principal causa genética o social), molecular, de los nervios, cognitivas y conductuales. Se hicieron las siguientes reivindicaciones principales:

"1. Que hay una fuerte genética en oposición a una causa social última para este trastorno. Los tipos de causas sociales propuestas (por ejemplo, abuso físico sexual en la infancia) debe elevar la capacidad de respuesta emocional, no dar lugar a la forma específica de la capacidad de respuesta reducida se ve en la psicopatía;

"2. La influencia genética conduce a la disfunción emocional que es el núcleo de la psicopatía;

"3. La influencia genética a nivel molecular sigue siendo desconocido. Sin embargo, parece afectar la integridad funcional de la amígdala y la corteza frontal orbital / ventrolateral y posiblemente sistemas adicionales;

"4. La interrupción dentro de estos dos sistemas neuronales conduce a un deterioro en la capacidad de formar asociaciones de estímulo-refuerzo y para alterar las asociaciones de estímulo-respuesta como una función de cambio de contingencia. Estos impedimentos interrumpen el impacto de las técnicas de socialización estándar y aumentan el riesgo de agresión reactiva, frustración inducida respectivamente".

Psicopatía y psicosis no son sinónimos. Psicópata y psicótico como sinónimos responden a un error o confusión recurrente en el uso coloquial de las palabras. La psicosis es una patología y la psicopatía un trastorno se podría decir "diametralmente opuestos" en una escala de empatía por comportar el primero su ausencia y el segundo por una exacerbación emocional. Salvada esta diferenciación lexicográfica, puede pasarse al análisis epidemiológico, y notar que a partir de la confrontación de los ítems de la PCL-R o Psychopathic Checklist Revisada y el PCL-SV o Psychopathic Checklist versión reducida para uso clínico que da origen al PCS o Psychopathy Criteria Set de Robert Hare (19), se presenta una correlación de la psicopatía con el trastorno de personalidad antisocial de 0.73; y con los criterios del CIE-10 para el Trastorno Disocial de Personalidad, de 0.79. Lo que quiere decir que sí hay una correspondencia importante.

\section{Criterios para el Diagnóstico del Trastorno Disocial.}

Implica un "patrón repetitivo y persistente de comportamiento en el que se violan los derechos básicos de otras personas 0 normas sociales importantes mentalmente coherentes con la edad. El sujeto tiene menos de 18 años. Puede tener un inicio infantil, antes de los 10 años, o inicio adolescente, después de los 10 años" (Asociación Americana de Psiquiatría, 1994); puede consultarse el novedoso trabajo de Blancharda, Lyonsa, et. al. (20).

\section{Trastornos de Personalidad con Diversas Formas de Violencia.}

\section{Psicopatía y Liderazgo para las Violencias:}

En una aproximación a la definición de psicopatía y el ejercicio del sadismo, León Mayer (21), sostiene:

"Se produce una idealización de un Sí Mismo patológicamente grandioso en términos de lo adecuado de la agresión bajo ciertas circunstancias y la capacidad de identificación con otras figuras poderosas también idealizadas como parte de grupos cohesionados, lo que permite que el sujeto desarrolle algún tipo de lealtad y la internalización de relaciones adecuadas con estas asociaciones. El psicópata, actuará motivado sólo por el deseo de poder y control sádico en un mundo que se divide entre los poderosos y los despreciables sin desarrollar lealtades de ninguna clase".

Jarman (22) advierte:

"No hay duda de que los psicópatas tienen éxito en las organizaciones y se elevan a los niveles superiores. Babiak, Neumann et al. (2010) encontraron que de una muestra de 203 líderes de negocios potenciales para el desarrollo directivo, nueve fueron considerados psicópatas. De los nueve dos eran vicepresidentes, dos eran directores, dos eran gerentes o supervisores y de aquí se celebró otro puesto de gestión; por tanto, que ya habían alcanzado su rango y condición considerable (p. 185). Hay tres grupos de razones por las que los psicópatas tienen éxito en las organizaciones: La primera es que las características personales de los psicópatas están orientados para solicitar y obtener el éxito. La segunda es que las organizaciones modernas buscan las características en las que se destacan los psicópatas. La tercera es que la dinámica de las organizaciones operan para mantener al psicópata en ese lugar de privilegio".

Babiak y Hare (23) también advierten: 
Óliver Lis

"Los políticos, anunciantes y vendedores no son los únicos que entienden que mirar y sonar bien a menudo ciega a la gente a lo que debería ser obvio para ellos: las distorsiones de la verdad, clichés vacíos, hipérboles, y fatuidad sin sentido".

\section{Borderline Personality Disorder o Trastorno Límite de la Personalidad y Violencias:}

Sinaia, Cave, et al. (24), advierten:

"Se ha propuesto que algunos subgrupos de pacientes con Borderline o Trastorno Límite de Personalidad serían más propensos a expresar el comportamiento violento (Nestor, 2002). Factores aparte de un diagnóstico de displasia broncopulmonar, tales como: duración de la estancia hospitalaria, el estilo de apego, el número de intentos de suicidio previos, la comorbilidad con otros trastornos de personalidad (trastorno de personalidad antisocial y psicopatía) y los trastornos del eje I con comorbilidad de abuso infantil y la expresión violencia en la infancia, se han propuesto aumentar el riesgo de violencia, sobre todo en poblaciones clínicas o forenses (Allen y Links, 2012)".

\section{Trastorno Negativista desafiante (oppositional defiant disorder) y Violencias:}

Murraya de Castro-Cerqueirab, et. al, (25) dicen:

"Se hicieron búsquedas en las siguientes bases de datos electrónicas para estudios elegibles de mayo de 2012: Social Science Citation Index, PubMed, y Lilacs (Lilacs es la mayor base de datos de la literatura científica en América Latina y el Caribe). Se utilizaron las siguientes palabras clave (y que también se tradujeron y se introdujeron en Lilacs por separado en portugués): [trastorno negativista u oposicionista desafiante, expresión de conducta o conductas problemáticas, crimen, violencia 0 delincuencia o de las drogas ilícitas o uso de sustancias 0 abuso de sustancias] y [cohorte prospectivo y longitudinal, y de estudios de control sobre la de la población elegida] y [prevalencia 0 velocidad 0 incidencia 0 frecuencia 0 factores de riesgo] y Brasil. A la lista de las referencias recuperadas mediante las búsquedas electrónicas, también hemos añadido los documentos de nuestros propios archivos, búsquedas en Internet, las recomendaciones de los investigadores brasileños, y artículos en las listas de referencias de los informes recuperados. Un diagrama de flujo del proceso de búsqueda y el cribado se muestra en la Fig. 1.

Figura $\mathbf{N}^{\circ} 1$.

Flujo del proceso de Búsqueda y el Cribado

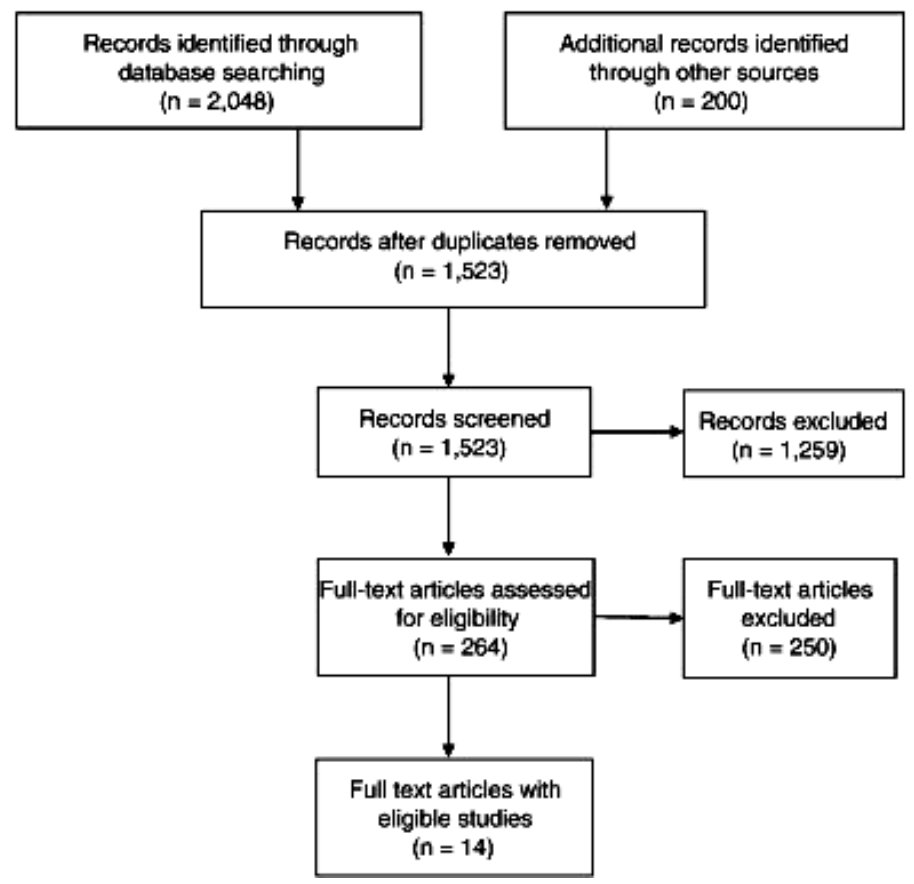

Fuente: Elaboración Propia 


\section{RESULTADOS:}

"Dos investigadores evaluaron de forma independiente los textos completos de elegibilidad. En la primera evaluación se hizo un tamizaje que eliminó el $88 \%$ de los artículos; se hizo una discusión sobre la pertinencia o no de los artículos restantes.

Los países de bajos y medianos ingresos en África y América tienen las tasas de homicidios más altas del mundo y soportan la mayor carga de la violencia en términos de años de vida ajustados por discapacidad (véase la Tabla 1). En 2008, la tasa de homicidios promedio mundial fue de 7,9 por cada 100.000 personas. La tasa en Brasil fue la tasa más alta con 29,6, quedando en el puesto 13 de los 193 estados miembros de la OMS. Entre 1980 y 2010, hubo un total de 1,09 millones de homicidios en Brasil. En 2004, se estima que 2,5 millones de años de vida sana se perdieron debido a la violencia $(2,488,000$ AVAD) en Brasil. Esta fue la mayor carga de los años de vida perdidos por la violencia en cualquier estado miembro de la OMS en ese año".

*La afirmación "los países de bajos y medianos ingresos en África y América tienen las tasas de homicidios más altas del mundo" no debe ser entendida metonímicamente, es decir, tomando los efectos por las causas: la psicopatía es causa de la violencia económica y de la desigualdad social de los pueblos. Dichos factores parecen tener una correlación con que la psicopatía se haga exponencial.

\section{Confluencia de Diversos Trastosnos y Violencias.}

\section{Sujetos pertenecientes a Grupos Armados llegales en Colombia}

Sobre la prevalencia de trastornos de personalidad en grupos armados, se ha comparado la significancia estadística de una escala de empatía para diversos trastornos de personalidad (trastorno disocial de personalidad, trastorno antisocial de personalidad que como ya se describió con pruebas de significancia, conforman psicopatía), relacionados con una misma forma de violencia: la violencia armada.

Tobón, Aguirre-Acevedo (26) presentaron una investigación desarrollada sobre una muestra pequeña de sesenta y tres excombatientes y 22 controles evaluados con WAIS (IQ), INECO(funciones ejecutivas), y un instrumento denominado Interpersonal Reactivity Index (Índice de Reactividad Interpersonal para medir empatía); junto a ello, el reconocimiento de las características emocionales y el MINI (perfil psiquiátrico).
Las implicaciones que tiene esta investigación sobre una muestra no muy significativa en términos poblacionales para efectos de validez y extrapolación de los resultados, no son tan importantes, como lo es que se haya demostrado una prevalencia de trastornos de personalidad en grupos armados colombianos al margen de la ley.

Sin embargo, se copian acá sus resultados:

"En comparación con el grupo control, los ex combatientes mostraron una mayor frecuencia de trastorno antisocial de la personalidad $(P=.031)$ y el trastorno disocial de comportamiento $(P=.017)$. En el perfil cognitivo, los ex combatientes mostraron una menor puntuación en la prueba de la función ejecutiva ( $M e=18.50 ; R Q=4,00$ ), control ( $\mathrm{Me}=23.00 ; R Q=5,25)$, con una angustia personal pobre en el perfil emocional $(\mathrm{Me}=10.00 ; \mathrm{RQ}=5,00)$ en comparación con el grupo control $(\mathrm{Me}=37.00 ; \mathrm{RQ}=$ $7,25)$ ".

La prueba $\mathrm{P}$ es una prueba de significación estadística de otra prueba, en este caso, una correlación de un perfil cognitivo, con un valor $P$ correspondiente a un perfil psiquiátrico emocional. De acuerdo al valor $\mathrm{P}$, ninguno de los valores es estadísticamente significativo:

Trastorno de Personalidad Antisocial antisocial personality disorder presenta una $\mathrm{P}=.031$;

Y trastorno de Personalidad de Comportamiento Disocial $(P=.017)$.

\section{Los Grupos llegales como Expresión Colectiva de Trastornos de Personalidad}

Ya tenemos una primera prueba de que hay presencia de trastornos de personalidad en grupos armados al margen de la ley en Colombia según Tobón, Aguirre-Acevedo (27). Vidal (28) nos ofrece una segunda prueba de esto tras aplicar la PCL-R de Hare validada en Colombia hace cerca de una década por García, Arango, et al. (29) y la Heider Simmel, prueba proyectiva, para convictos de las FARCEP.

Según Hare (30) el 1\% de la población mundial es psicópata, con prevalencia de entre $15 \%$ y $25 \%$ en cárceles (es decir, ese 1\% hace un daño por entre un 15\% y un $25 \%$ ), ascendiendo hasta un $40 \%$ en su prevalencia dentro de la banca, la política, los hospitales y los grupos armados legales e ilegales.

No se ha hecho un estudio aún de prevalencia de otros trastornos en Colombia, aparte de los mencionados que figure en estas bases de datos; lo que denota una necesidad de convertirse en un gran laboratorio de paz, de 


\section{Óliver Lis}

estudios de este cariz, por su remota y persistente violación a los derechos humanos, su corrupción, sus desigualdades, su remoto y persistente atraso, sus revoluciones incesantes y la demanda de políticas eficaces para un actual proceso de paz.

Según Babiak y Hare, (31) los psicópatas pues, pueden llegar a organizarse para conseguir sus fines de poder, dejando en su carreara toda una estela de violencias (no necesariamente físicas) y destrucción (no necesariamente física, sino moral), con prontuario o sin él.

Según los trabajos científicos consultados, las bandas delincuenciales y los grupos armados al margen de la ley también pueden ser considerados como una expresión colectiva de diversos trastornos de personalidad, tal como lo sostiene Jarman (32).
Otros trastornos de personalidad como el borderline o el trastorno negativista desafiante, pueden unirse voluntariamente a la causa de estos grupos para expresar su violencia contra la autoridad materna o paterna proyectada en formas de autoridad estatales 0 institucionales, razón de afinidades ideológicas, culturales, intelectuales, etc., pudiendo tener roles distintos e incluso ajenos a la vocación de poder, dentro de una misma organización delincuencial, según las capacidades del sujeto, su educación, su historia personal (con pérdida del control infantil o sin él) y las características del trastorno. Esto también puede legitimarse por las vías democráticas 0 de la violencia institucional, pues hay carreras como las armadas o las que tienen vocación de poder, como la del político, que pueden resultar la profesionalización de un síntoma como la pérdida del control infantil que busca recuperarse a través del poder, del dinero y del mando.

Figura $\mathrm{N}^{\circ} 2$.

Pirámide jerárquica de Óliver Lis de trastornos de personalidad en organizaciones armadas.

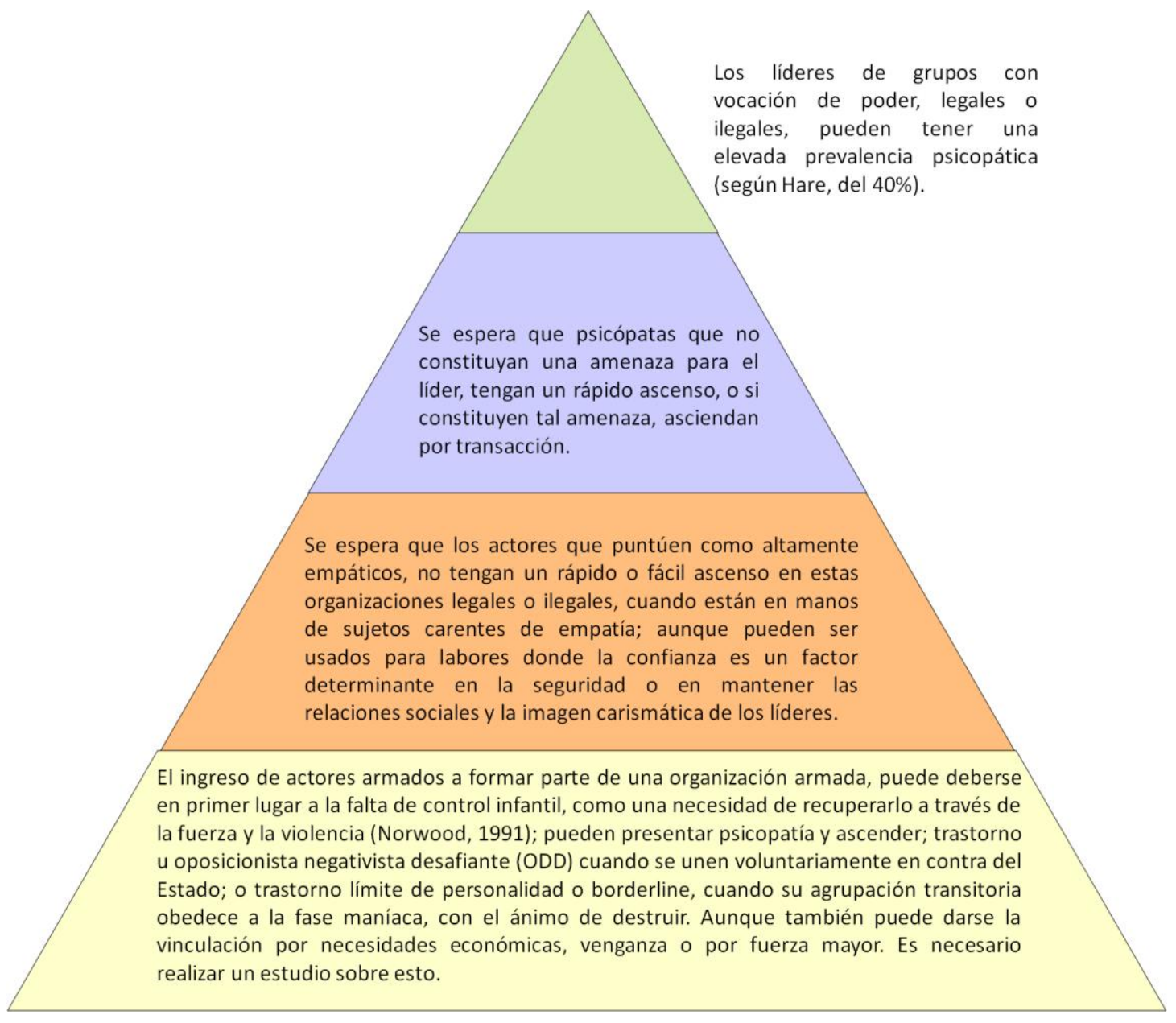

Fuente: Elaboración Propia 
Los sujetos que delinquen contra el patrimonio económico de una ciudad, contra la salud, o que depredan los rubros destinados para atender necesidades de poblaciones vulnerables (lo que es frecuente en Colombia), tendrán una mayor prevalencia psicopática en ámbitos o escenarios como el de la política, por sobre otros grupos que no presentan una clara vocación de poder; las desigualdades sociales de un país parecen ser directamente proporcionales a la prevalencia de trastornos de personalidad que llegan a organizarse dando origen a las violencias.

Variables que podrían interactuar con la biología en el surgimiento de los trastornos de personalidad que implican falta de empatía y a su vez determinan el ingreso de estos sujetos a estos grupos, pueden ser los contemplados en los Índices de Desarrollo Humano (que no deben confundirse con el PIB aunque dependan parcialmente de este); el nivel de desarrollo moral de acuerdo a PérezDelgado y Maestre (33), pruebas diagnósticas para trastornos de personalidad que comprometen la empatía como la PCL-R de Hare o la Heider Simmel, conforme a los trabajos de Tobón (34) et. al., Vidal (35) León Mayor, etc.; a genética, a través de estudios de cariotipo humano, de acuerdo a las investigaciones de Blancharda, Lyonsa, et. al. (36), Morris, Skuse et al. (37), Lake, Baksh, et al. (38), Kessler y Moos (39), como criterios que deberían tenerse en cuenta para un nuevo censo nacional.

La ausencia de empatía es el elemento común en las descripciones de los manuales de diagnóstico para estos trastornos que comprometen violencias (DSMV-R, CIE-10 y OMS), por ello, se sugiere generar una escala de empatía universal como base para trabajar sobre el diagnóstico de los diferentes trastornos.

Las demás causas de la violencia armada, como la violencia económica, también podrían reducirse sensiblemente cuando sean tomadas las medidas adecuadas para evitar que los sujetos diagnosticados con estos trastornos puedan ingresar a cargos administrativos, representativos, de responsabilidad, al servicio del público, reduciéndolos a labores bajo un estricto control donde no se comprometa la integridad de las demás personas y de la sociedad, mientras se considera la posibilidad del aborto legal para fetos que vengan con trisomía en el cromosoma sexual, mientras la ciencia no pueda corregir estos problemas genéticos y neurológicos interviniendo el cerebro humano 0 descubriendo otras formas de contrarrestar la epidemia.

Es posible tener con estas medidas preventivas y con una educación para la empatía, un cambio definitivo sobre el tipo de sistemas económicos, políticos, sociales, jurídicos, y hasta de parentesco que se desarrollan en la actualidad, pues el desequilibrio violento de tales sistemas, parece ser la expresión de trastornos de personalidad colectivos, fijados en la historia y en sus prácticas culturales.

Finalmente, el estado de desarrollo moral posconvencional propuesto por Laurence Kohlberg (40) entendido como una conciencia capaz de refrenar impulsos violentos, sexuales, de no infligir algún tipo de sufrimiento sobre otros sin la presencia de normatividad que lo premie o castigue parece obedecer a un nivel de alta empatía, al cual no se puede llegar a través de la educación, pero sí se puede hacer un proceso para que los sujetos que no presentan trastornos que desemboquen en violencias, puedan pasar de un estado de desarrollo moral preconvencional a un estado de desarrollo moral convencional, es decir, que si no pueden dejar de hacer el daño por un criterio propio, al menos acepten las normatividad (lo que es imposible en los psicópatas).

Un ejemplo de esto puede ser consultado en la citada obra de Pérez-Delgado y Maestre (cita 33), reconociendo la incapacidad de lograr a través de su intervención educativa, el desarrollo moral posconvencional esperado (ver resultados de la ejecución de la TSCS, Self-Concept Scale de estos autores).

Esta conclusión es importante, por cuanto se entiende que ni la educación, ni ningún factor externo como una nueva Asamblea Nacional Constituyente, podrán ponerle fin al fenómeno de la violencia, si se demuestra que depende plenamente de los trastornos de personalidad organizados, lo que resulta bastante posible.

Puede ser por ello que la Nación colombiana que ha contado con varias Asambleas Constituyentes desde su fundación como República en 1819, como Estado Federado en 1863, como República nuevamente en 1886, y como otra República aparentemente más incluyente desde 1991 hasta la actualidad, entre otras asambleas constitucionales intermedias, no haya superado el flagelo del conflicto armado, muy a pesar de que su Carta Constitucional más reciente contempla la creación de un Estado Social de Derecho con un trato desigual para situaciones desiguales, la presentación de los derechos fundamentales como el derecho a la vida, a la salud, a la educación, al trabajo, la dignidad humana, una nueva Corte Constitucional de modelo anglosajón dentro del viejo modelo francés del Civil Law, en medio de la contradicción que supone toda Asamblea Nacional, como pacto entre fuerzas. 


\section{CONCLUSIONES:}

De acuerdo a los trabajos analizados, en Colombia hay prevalencia de estos trastornos de personalidad en una relación con diferentes formas de violencia, como lo confirman los trabajo de Vidal (ver citas 13, 28, 35) y Tobón, Aguirre-Acevedo et al. (41).

Los trastornos de personalidad como el trastorno disocial y trastorno antisocial, tienen una alta significancia $(\mathrm{P})$, con la psicopatía, por lo que se puede concluir que hacen parte de la misma y tienen, según los estudios de Tobón, Aguirre-Acevedo (42). Carlos Vidal (43), una relación con la violencia armada en Colombia.

Conforme al trabajo de Murraya de Castro-Cerqueirab, et. al, (44), el trastorno negativista u oposicionista desafiante, también puede involucrarse en violencias y violencia armada.

Desde la investigación de Sinaia, Cave, et al._(45), también puede concluirse que el trastorno límite de la personalidad 0 borderline, puede presentar comorbilidad psicopática y ser sin ella causa de violencias y de violencia armada.

Según Tobón, Aguirre-Acevedo (46), se presenta comorbilidad entre varios de estos trastornos que confluyen en expresiones violentas y de violencia armada en el caso colombiano.

De acuerdo a Jarman (47), Babiak y Hare (23), las organizaciones delictivas, legales e ilegales, también pueden ser la expresión colectiva de trastornos de personalidad como la psicopatía.

El trabajo de Blair (47), a tono con los de Blancharda, Lyonsa, et. al. (48), Morris, Skuse et al. (49), Lake, Baksh, et al. (50), Kessler \& Moos (51), permite colegir que no está muy lejana una definición de enfermedad para la psicopatía y otros trastornos de personalidad afines que comportan violencia; también que la homogenización y estandarización de su diagnóstico y tratamiento, facilitará un metaanálisis para trabajar los trastornos de personalidad y su relación con las diversas formas de violencia, lo que permitirá formular políticas más eficaces para erradicar el flagelo de la guerra.
Es preciso generar un diagnóstico epidemiológico y generar un debate sobre el tratamiento de aquellos que por cariotipo han sido identificados antes de nacer como psicópatas, así como a esta clase de sujetos con esta patología incurable que constituyen una amenaza para la vida y para la paz.

De acuerdo a Pérez-Delgado y Vicenta Maestre (52), el contrato social con sus garantías constitucionales, los diversos sistemas políticos, sociales, económicos, la monarquía, la democracia, los totalitarismos, ya sean de izquierda 0 de derecha, los derechos humanos y la educación, no constituyen herramientas suficientes para erradicar las causas de los trastornos de personalidad que comprometen violencia como la psicopatía, y que tienen un origen genético, pero ayudan a que individuos que no presentan trastornos de personalidad, puedan acatar las normas de un Estado (es decir, para que pasen de un estado preconvencional del desarrollo moral según Kohlberg(53), a un estado convencional, pese a que no puedan lograr el estado ideal del desarrollo moral posconvencional, que denota un elevado nivel de empatía, como podemos colegir de la obra de Pérez-Delgado y Maestre (54).

Variables que podrían interactuar con la biología en el surgimiento de los trastornos de personalidad que implican falta de empatía y a su vez determinan el ingreso de sujetos a grupos armados al margen de la ley, pueden ser los contemplados en los Índices de Desarrollo Humano (que no deben confundirse con el PIB, aunque dependan parcialmente de este). Bajo nivel de desarrollo moral en la escala adaptada por Pérez-Delgado y Maestre (55) de acuerdo a Kohlberg (56); pruebas diagnósticas para trastornos de personalidad que diagnostican psicopatía como la PCL-R de Hare o la Heider Simmel, conforme a los trabajos de Tobón (57) et. al., Vidal (58), León Mayor (59), etc.; y especialmente la genética, a través de estudios de cariotipo humano, de acuerdo a las investigaciones de Blancharda, Lyonsa, et. al. (60), Morris, Skuse et al. (61), Lake, Baksh, et al. (62), Kessler \& Moos (63), para determinar trisomía en fetos, como criterios que deberían tenerse en cuenta para un nuevo censo nacional orientado al Posconflicto. Si el Registro Civil de Nacimiento incluyera el cariotipo humano según fue propuesto por el suscrito autor (64) y se tomaran medidas al respecto, quizá podría erradicarse en un par de generaciones el flagelo de la guerra. 


\section{REFERENCIAS:}

Abraham J, Rahardjo W. Psychopathy, sexual values dimensions, and premarital sexual behavior among urban unmarried adolescents, Procedia - Social and Behavioral Sciences[Internet]. 2015 [consultado 1 de enero de 2016]; 165: 2-11. Disponible en: http://ac.els-

cdn.com/S1877042814067378/1-s2.0-

S1877042814067378-

main.pdf?_tid=fe159e90-1aec-11e6-b295-

00000aab0f02\&acdnat $=1463351765 \_82 \mathrm{e} 03$

8d850617a71fc73c2f18772ac93

Atarhouch, N.; Hoffmann, E; Adam, S.; Titeca, J., Stillemans, E.; Possion, P.; Le bon, O.; Servais, L.; Évaluation des traits caractéristiques de la psychopathie chez les adolescents délinquants. Evaluation of typical psychopathic traitswith juvenile offenders, L'Encéphale, Volume 30, Issue 4, September [Internet]. 2004 [consultado 26 de mayo de 2016], P. 369-375.

Babiak, Paul, Ph.D., Hare, Robert, Ph.D., "Snakes in Suits, When Psychopaths Go to Work", HarperCollins Publishers Inc., [Internet]. 2007 [consultado 26 de mayo de 2016] (23, 31).

Blair, Peschardt, Budhani, Mitchell, Pine, "Thedevelopment of psychopathy", J ChildPsycholPsychiatry, Mar-Apr, [Internet]. 2006 [consultado 20 de mayo de 2016] (18, 47)

Borenstein, M., "Meta-Analysis: Concepts and Applications", SeminarStatisticalHorizons, [Internet]. 2013.

Blancharda, Alyson; Lyonsa, Minna; Centifantib, Luna, Baby was a black sheep: Digit ratio (2D:4D), maternal bonding and primary and secondary psychopathy, personality and individual differences; volume 99 , september 2016, p. 67-71, University of Liverpool, Durham University, United Kingdom, [Internet] $2016(4,9,20,36,48,60)$ [consultado 26 de mayo de 2016].

Cortina, Adela, Neuroetica y Neuropolitica, Sugerencias para la Educacion Moral. Technos, 2011.
García Valencia, Jenny, Arango Viana, Juan Carlos, Correa Rico, Oscar, Pérez González, Andrés Felipe, Agudelo, Víctor Hugo, Mejía Mosquera, Carlos Andrés, Casals, Sergi, López Calle, Gabriel Jaime, Patiño López, Juan David, Palacio Acosta, Carlos

Alberto, "Validación de la Lista de Chequeo de Psicopatía-Revisada (PCL-R) en poblacióncarcelaria masculina de Colombia", Revista Colombiana de Psiquiatría, [Internet]. 2008 [consultado 1 de enero de 2016] (29). Disponible en: http://www.scielo.org.co/scielo.php?script=sc i_arttext\&pid=S0034-74502008000400007

Gustavsson, A.; Svensson, M; Jacobi, F; Allgulander, C; Alonso, J.; Beghi, E.; Dodel, R.; Ekman, M; Faravelli, C., Fratiglioni, L., Gannon, B.; Jones, D.H.; Jennum, P.; Jordanova, A, Jönsson, L, Karampampa, K, Knapp, M, Kobelt, G, Kurth, T, Lieb, R, Linde $M$, Ljungcrantz C, Maercker A, Melin B, Moscarelli M, Musayev A, Norwood F, Preisig M, Pugliatti M, Rehm J, SalvadorCarulla L, Schlehofer B, Simon R, Steinhausen HC, Stovner LJ, Vallat JM, Van den Bergh $\mathrm{P}$, van Os J, Vos $\mathrm{P}, \mathrm{Xu} \mathrm{W}$, Wittchen HU, Jönsson B, Olesen J; CDBE, 2010 Study Group, Cost of disorders of the brain in Europe 2010. Eur Neuropsychopharmacol. $2011 \quad$ Oct; 21(1):718-79. Epub [Internet] 2011 [consultado 1 de junio de 2015]. Disponible en:

http://www.ncbi.nlm.nih.gov/pubmed/219245 89

Hare, Robert. "Human Psychopathy and the DSM-IV Criteriafor Antisocial Personality Disorder". Journal of Abnormal Psychology, by the American Psychological Association, [Internet]. August 1991 [consultado 1 de junio de 2015] $(16-17,19,30)$ Vol. 100, No. 3, 391-398. Disponible en: http://psych.utoronto.ca/users/peterson/psy4 30s2001/Hare\%20RD\%20Psychopathy\%20J AP\%201991.pdf 
Óliver Lis

Hare, R. D., "La naturaleza del psicópata: Algunas observaciones para entender la violencia depredadora humana". En A. Raine, \& J. Sanmartín (Eds.), "Violencia y Psicopatía" (2a. ed.). España: Ariel, 2000 [consultado 1 de julio de 2015], p. 17-49.

Heller, John h. "Human ChromosomeAbnormalities as RelatedtoPhysical and Mental Dysfunction". Perspectives in AbnormalBehavior, 1974 [consultado 22 de agosto de 2015], P. 214230.

"Human Afflictions and ChromosomalAberrations", EN International Series of Monographs in Pure and AppliedBiology: Modern Trends in PhysiologicalSciences, 1969 [consultado 22 de mayo de 2015], P. 385-392.

Jarman, Rod, "Psychopathy as a Phenomenon of Interest in InformationSystemsResearch", Proceedings of the 21st EuropeanConferenceonInformationSystems, CurtinUniversity, [Internet] 2013 [consultado 2 de enero de 2016] $(22,32,47)$. Disponible en:

http://www.sciencedirect.com/science/article/ pii/S0165178115001559

Kessler, Seymour, Moos, Rudolf $H$. "The XYY karyotype and criminality: A review". Journal of PsychiatricResearch, Volume 7, Issue 3, [Internet]. February 1970 [consultado 26 de mayo de 2015], (7, 12, 29, 51, 63) P. 153170.

KOHLBERG, Lawrence, "Moral stages and moralization: The cognitive-develop, mental approach. Moral Development and Behavior: Theory, Research and Social Issues", 1987 $(40,53,56)$.

Lake, C. Raymond, Baksh, H., Rozana, Wiedeking, Claus, Chernow, Bart, Owen, Walter W., Ziegler, Michael G., Sympatheticnervoussystemfunction in XYY subjects. Original Research Article. Psychiatry Research, Volume 9, Issue 2, [Internet] June 1983(6, 11, 38, 50, 62) [consultado 2 de junio de 2015], P. 149-155.

León Mayer, Elizabeth M.,"La psicopatía en prisioneros chilenos: prevalencia y métodos de evaluación", Universidad Nacional de La
Plata, tesis doctoral; [Internet]. 2012 [consultado 11 de mayo de 2016] (21). Disponible en: http://sedici.unlp.edu.ar/bitstream/handle/109 15/25394/Documento_completo.pdf?sequen $\mathrm{ce}=1$

León Mayer, Elizabeth M., Folino, Jorge, Neumann, Craig, Hare, Robert, "Theconstruct of psychopathy in a Chileanprisonpopulation", Revista Brasileira de Psiquiatría de la Asociación Brasilera de Psiquiatría [Internet] 2015 [consultado 11 de mayo de 2016]: Disponible en: http://www.scielo.br/pdf/rbp/2015nahead/151 6-4446-rbp-1516-4446-2014-1540.pdf

Macdougall, Joyce. Teatro Transicional. Ed. Trejos, Barcelona [Internet], 2006 [consultado 11 de mayo de 2016].

Memorias Gencauca. VII Congreso Nacional de Genealogías. Lis, Óliver, Genealogías Modernas y Premodernas. Nuevos Retos del Oficio Genealógico (sobre la aplicación de pruebas de cariotipo para poner las genealogías al servicio de las ciencias de la salud). Armenia, Colombia, 14 y 15 de noviembre de 2015.

Ministerio de Salud de Colombia, Colciencias, "Encuesta Nacional de Salud Mental", Javegraf, Bogotá; [Internet]. 2015 [consultado 6 de mayo de 2016].

Morris, J.; Skuse, D.H.; Dolan, R.J.; S.11.03 Fear, amygdala and the $\mathrm{X}$ chromosome; European Neuropsychopharmacology, Volume 13, Supplement 4, October [Internet]. 2003 [consultado 6 de mayo de 2016] $(5,10,37$, $49,61)$ S124-S125.

Murraya, Joseph; De Castro-Cerqueirab, Daniel Ricardo, Kahnc, Tulio, "Crime and Violence in Brazil: Systematic Review of Time Trends, Prevalence Rates and Risk Factors", Aggression and Violent Behavior, Volume 18, Issue 5, September-October [Internet]. 2013 [consultado 6 de mayo de 2016] (25, 44), 471-483. Disponible en: http://www.sciencedirect.com/science/article/ pii/S0193397316300028

Pera-Guardiola, Contreras-Rodríguez, Batalla, Kosson, Menchón, Pifarré, Bosque, Cardoner, Soriano-Mas, "BrainStructuralCorrelates of EmotionRecognition in Psychopaths", 
PubMed, [Internet]. 2016 [consultado 11 de mayo de 2016].

Pérez-Delgado y Maestre, Vicenta, Cognición y afecto en el desarrollo moral: evaluación y programas de intervención, Ed. Ariel, Barcelona, 1999 (33, 52, 54).

Schumanna, Gunter; Binderb, Elisabeth B., ArneHoltec, E. Ronald de Kloetd, Ketil J. Oedegaarde, Trevor W. Robbinsf, Tom R. Walker-Tilleya, IstvanBitterao, Verity J. Browng, JanBuitelaarh, Roberto Ciccocioppoi, RoshanCoolsj, Carles Escerak, Wolfgang Fleischhackerl, HertaFlorm, Chris D. Frithn, Andreas Heinzo, Erik Johnsene, Clemens Kirschbaump, TorkelKlingbergq, Klaus-Peter Leschr, ShonLewiss, Wolfgang Maiert, Karl Mannu, Jean-LucMartinotv, w, Andreas MeyerLindenbergm, Christian P. Müllerx, Walter E. Müllery, David J. Nuttz, Antonio Persicoaa, Giulio Perugiab, MathiasPessiglioneac, Ulrich W. Preussad, Jonathan P. Roiserae, Paolo M. Rossiniaf, Janusz K. Rybakowskiag, Carmen Sandiah, Klaas E. Stephanai, Juan Undurragaai, EduardVietaaj, Nic van der Weeak, TilWykesal, Josep MariaHaroam, Hans UlrichWittchenan, "Stratified medicine for mental

EuropeanNeuropsychopharmacology,

Volume 24, Issue 1, [Internet]. January 2014

$(2,15)$ [consultado 3 de diciembre de 2015], P. 5-50. Disponible en: http://www.sciencedirect.com/science/article/ pii/S0924977X13002769

Sinaia, Cave, Hirvikoskib, Tatja, Nordströma, AnnaLena, Nordströma, Peter, Nilsonnea, Wilczeka, Åsa Alexander, Åsbergc, Åsbergc, Jokinena, Jussi, "Thyroid hormones and adult interpersonal violenceamongwomenwithborderlinepersonal itydisorder". PsychiatryResearch, Volume 227, Issues 2-3, 30 [Internet]. June 2015 [consultado 17 de octubre de 2015] $(24,45)$, 253-257. Disponible en: http://www.sciencedirect.com/science/article/ pii/S0165178115001559

Titchener, E.B., "Introspection and empathy Dialogues in Philosophy", Mental and NeuroSciences, pág. 25-30, [Internet]. 19092014 [consultado 10 de enero de 2015]. Disponible
http://www.crossingdialogues.com/Ms-E1401.pdf

Tobón, Aguirre-Acevedo, Velilla, Duque, Ramos, Pineda, "Psychiatric, Cognitive and EmotionalProfile in Ex-combatants of IllegalArmedGroups in Colombia", Revista Colombiana de Psiquiatría, Jan-Mar, 2016 [consultado 11 de mayo de 2016], (26-27, $34,41-42,46,57) 28-36$.

Turpin, Raymond, LEJEUNE, Jérôme, "Human Afflictions and ChromosomalAberrations", CHAPTER $\quad 10$

NumericalGonosomalAberrations, [Internet]. 1969 (3, 8) [consultado 27 de mayo de 2015], P. 184-214. Disponible en: http://www.sciencedirect.com/science/book/9 780080132631

Vidal Reyes, Carlos Alberto, "Criminalidad, psicopatía y funcionamiento de la corteza prefrontal", Tésis de Maestría, Universidad del Valle, [Internet]. 2014 [consultado 28 de junio de 2015] $(13,28,35,43,58)$.

Vygotski, Lev, "Pensamiento y Lenguaje", Edición virtual, 1934.

Völlm, Birgit, Taylor, Alexander; Richardson, Paul; Corcoran, Rhiannon; Stirling, John; McKie, Shane; Deakin, John F.W.; Elliott, Rebecca; Neuronal correlates of theory of mind and empathy: A functional magnetic resonance imaging study in a nonverbal task, Neurolmage \# 29, [Internet] 2006 [consultado 1 de enero de 2016]. (14) 9098. Disponible en: http://www.christofflab.ca/pdfs/2009/01/vollm -et-al-2006.pdf

Bases de Datos de Artículos Científicos:

http://lilacs.bvsalud.org/es/

http://www.hare.org/links/media.html

http://sedici.unlp.edu.ar/

http://www.sciencedirect.com/

http://www.ncbi.nlm.nih.gov/pubmed

http://thomsonreuters.com/ 
Investigaciones de Fuentes Oficiales:

World Health Organization (WHO). "Mental Health Action Plan 2013-2020", Geneva; 2013. Disponible en: http://apps.who.int/iris/bitstream/10665/89966/1/97892 41506021_eng.pdf?ua=1

"Salud mental: fortalecimiento de nuestra respuesta". WHO. $2014 . \quad$ Disponible en: http://www.who.int/mediacentre/factsheets/fs220/es/

Ley 1616 de 2013 de salud mental. República de Colombia. Disponible en: http://wsp.presidencia.gov.co/Normativa/Leyes/Docum ents/2013/LEY\%201616\%20DEL\%2021\%20DE \%20E NERO\%20DE\%202013.pdf

Ministerio de Salud y Protección Social (MSPS). Sistema de Monitoreo y Evaluación al Plan Decenalde Salud Pública 2012-2021 [Internet]. Bogotá; 2013. Disponible en: http://www.minsalud.gov.co/Documentos $\% 20 \mathrm{y} \% 20 \mathrm{Pu}$ blicaciones/Sistema $\% 20 \mathrm{de} \% 20$ Seguimiento $\% 20 \mathrm{y} \% 20$ Evaluaci\%C3\%B3n\%20del\%20Plan\%20Decenal\%20De\%20Salu d $\% 20 \mathrm{P} \%$ C3\%BAblica $\% 20$ -

\%20PDSP\%20Colombia\%202012\%20-\%202021.pdf.

Asociación Colombiana de Psiquiatría, Comité de Políticas. http://www.psiquiatria.org.co/index.html

Poltítica Nacional del Campo de la Salud Mental. Documento-Propuesta para Discusión y Acuerdos. Bogotá: Kimpres; 2007.

Disponible en: http://psiquiatria.org.co/web/wpcontent/uploads/2013/05/Pol\%C3\%ADtica-Nacionaldel-Campo-de-la-Salud-Mental-versi\%C3\%B3n-final2.pdf

Colegio Colombiano de Psicólogos. Normatividad: http://www.colpsic.org.co/quienes-

somos/normatividad/20

\section{Leyes}

República de Colombia. Congreso de Colombia. La ley 1616 de 2013, enero 21, por la cual se reglamenta la Salud Mental en Colombia y se dictan otras disposiciones. Diario Oficial No. 48680. Bogotá: El Ministerio; 2013.
República de Colombia. Congreso de Colombia. La ley 1616 de 2013, junio 10, por la cual se reglamenta la Atención a Desplazados en Colombia y se dictan otras disposiciones. Diario Oficial No. 48.096. Bogotá: El Ministerio; 2011. 